\title{
Intervenciones psicológicas en el tratamiento de la ansiedad en personas con cáncer de mama: un meta-análisis
}

\author{
Psychological Interventions in Anxiety Treatment in \\ People with Breast Cancer: a Meta-Analysis
}

\author{
Susana Zelaya-Rivas ${ }^{1}$ \\ ${ }^{1}$ Escuela de Psicología, Universidad de Costa Rica, Costa Rica
}

Resumen. Objetivo. Esta investigación busca estimar el tamaño del efecto de las intervenciones psicológicas para el tratamiento de la ansiedad en personas con cáncer de mama. Método. Se realizó una revisión sistemática de bases de datos en línea (Dialnet, Ebsco, Latindex, PubMed, ScienceDirect, Wiley, SAGE, Scielo y Google Scholar), a través de la cual se extrajeron artículos experimentales y cuasi-experimentales relevantes para el tema de estudio. De los artículos seleccionados, se extrajeron datos de las participantes, los tratamientos utilizados y datos pretest y postest de los grupos para el análisis. Resultados. Diecisiete artículos que cumplieron los criterios de inclusión fueron agregados en el análisis. El tamaño de efecto global de las intervenciones fue de $(g=-.459)$. Asimismo, se realizaron análisis de sesgo y de variables continuas moderadoras.

Palabras clave. Ansiedad, cáncer de mama, intervenciones psicológicas, meta-análisis, revisión sistemática.

Abstract. Objective. This study aims to estimate the effect size of psychological interventions for anxiety treatment in people diagnosed with breast cancer. Method. A systematic review of online databases (Dialnet, Ebsco, Latindex, PubMed, ScienceDirect, Wiley, SAGE, Scielo, and Google Scholar) was carried out, through which experimental and quasi-experimental articles relevant to the analysis were retrieved. From the selected articles, data were extracted regarding the participants, the treatments used, and pre and post test data from the groups for analysis. Results. Seventeen articles that met the inclusion criteria were used in the analysis. The overall effect size of the interventions was determined $(g=-.459)$. Likewise, bias and continuous moderating variables analyses were performed.

Keywords. Anxiety, Breast cancer, Psychological interventions, Meta-Analysis, Systematic review. 


\section{Introducción}

El diagnóstico de cáncer de mama presenta retos importantes para la persona que lo recibe, en diversas áreas de su vida. La enfermedad, los eventos que transcurren tras el diagnóstico y los tratamientos que suelen aplicarse pueden tener efectos importantes en la persona, tanto a nivel físico con síntomas como dolor, náuseas, debilidad, fatiga y disfunción en el sueño, como a nivel psicosocial con presencia de estrés, ansiedad, miedo de recurrencia, problemas familiares, depresión, dificultades económicas y disminución en la calidad de vida, incluso tras la finalización del tratamiento (Huang \& Shi, 2016; Smith-Turchyn, Morgan, \& Richardson, 2016; Zhang et al., 2015; Zhang, Xu, Wang, \& Wang, 2016).

Ahora bien, la ansiedad se ha determinado como una de las consecuencias más comunes en pacientes y sobrevivientes de cáncer de mama, con presencia en hasta un $60 \%$ en este grupo, un porcentaje más elevado que el de la población general y el de pacientes con otros tipos de cáncer (Huang \& Shi, 2016). Los síntomas de ansiedad se pueden presentar antes, durante y después de los tratamientos y tienen un efecto negativo en los sistemas de respuesta neuroendocrinos, lo que puede llevar a perturbaciones inmunológicas que contribuyen, a su vez, a la recurrencia o permanencia de la enfermedad (Huang \& Shi, 2016; Zhang et al., 2015).

En este sentido, la ansiedad, que incluye los síntomas de miedo excesivo e irracional, sentimientos tensos y dificultad para manejar tareas diarias o el malestar causado por estas tareas, tiene un efecto importante en el estado general de la persona (Rector, Bourdeau, Kitchen, \& Joseph-Massiah, 2008). Cierto nivel de ansiedad es necesario y adaptativo, pues permite emitir conductas de afrontamiento a situaciones de peligro; sin embargo, puede volverse dañina cuando la activación es excesiva, pues interfiere con la capacidad de manejar las situaciones demandantes y las actividades cotidianas, y puede afectar la salud y el bienestar (Lim, Devi, \& Ang, 2011; Rector et al., 2008).

Como plantean Rector et al. (2008), cuando la respuesta de pelea o huida se activa, hay tres elementos que se ponen en funcionamiento: el cognitivo, el conductual y el fisiológico. De esta forma, en el aspecto cognitivo la atención se fija de manera inmediata en la amenaza, en el conductual se producen comportamientos y se omiten algunos con el propósito de protegerse (como evitar ciertas situaciones o actividades), y en el fisiológico se encuentran síntomas como respiración superficial, temblores, mareos, sudoración, palpitaciones, tensión muscular y náuseas.

En este sentido, se ha planteado la necesidad de intervenciones efectivas, no farmacológicas (debido a la gran cantidad de medicamentos que suelen prescribirse ante un diagnóstico de cáncer), que permitan manejar estos efectos negativos y que faciliten la mejora de la calidad de vida de pacientes y sobrevivientes de cáncer de mama (Smith-Turchyn et al., 2016; Zhang et al., 2016). Así, la implementación de intervenciones psicológicas permite el manejo de estas manifestaciones psicosociales de la enfermedad, con el objetivo de reducir la ansiedad y la tensión lo que, a su vez, se relaciona con mejores resultados del tratamiento y facilita la reducción del dolor asociado (Zhang et al., 2015).

De esta forma, se evidencia que las intervenciones psicológicas deben utilizarse como un componente importante de apoyo, con el fin de reducir la mortalidad, la morbilidad 
y el sufrimiento en esta población, desde el momento del diagnóstico hasta la remisión, e incluso en fases terminales (Huang \& Shi, 2016). Los cambios que presentan la enfermedad y el tratamiento requieren de suficiente apoyo social y estrategias de afrontamiento, elementos que desde la intervención psicológica también pueden ser trabajados y facilitados (Richter et al., 2015).

Al tomar en cuenta la multicausalidad en el desarrollo del cáncer, la necesidad de tratamientos psicológicos eficaces que favorezcan la salud integral y el bienestar de la persona, y la prevalencia elevada de este tipo de cáncer, se intensifica la necesidad de conocer qué tratamientos psicológicos son eficaces en el manejo de la ansiedad, de gran prevalencia en esta población. El cáncer de mama es el segundo cáncer más frecuente y el primer tipo de cáncer como causa de muerte en mujeres en Costa Rica (Instituto Nacional de Estadística y Censos de Costa Rica, 2017; Ministerio de Salud de Costa Rica, 2014); asimismo, es el tipo de cáncer más frecuente en mujeres alrededor del mundo y el mayor causante de muertes por enfermedad oncológica en las mujeres (Organización Mundial de la Salud, 2020). De este modo, resulta necesario conocer no únicamente qué tipo de tratamientos suelen utilizarse en estos casos, sino cuáles han mostrado resultados sólidos con base en la evidencia disponible.

Así, esta investigación se planteó como un meta-análisis que permitiera conocer el tamaño del efecto global de las intervenciones psicológicas en el tratamiento de la ansiedad en personas con cáncer de mama. El meta-análisis, como estrategia metodológica, constituye una revisión sistemática que utiliza técnicas estadísticas para el análisis cuantitativo de los resultados de estudios sobre un tema específico (Marín, Sánchez, \& López, 2009).

Como explica Giménez (2012), los meta-análisis permiten la combinación matemática de los resultados de diversas investigaciones con el fin de contestar una problemática específica. De acuerdo con esta autora, entre los objetivos que permiten alcanzar se encuentra contar con una guía que permita la toma de decisiones, brindar una respuesta a una pregunta que no se ha podido resolver por la presencia de resultados diversos en las investigaciones llevadas a cabo, analizar el nivel de heterogeneidad de los resultados de las investigaciones y estimar el tamaño del efecto de una intervención específica, como es el caso del presente estudio. De esta forma, el meta-análisis se constituyó como una estrategia apropiada para alcanzar el objetivo de la investigación, a saber, estimar el tamaño del efecto de las intervenciones psicológicas para el tratamiento de la ansiedad en personas con cáncer de mama.

Ciertos estudios meta-analíticos han realizado aproximaciones al estudio de la efectividad de las intervenciones psicológicas sobre la salud mental de personas con cáncer. Así, Sheard y Maguire (1999) hicieron dos meta-análisis de ensayos clínicos que aplicaran intervenciones psicológicas tanto grupales como individuales, para la prevención o tratamiento de la depresión y la ansiedad en personas con cáncer. La depresión fue la medida tomada en cuenta en veinte estudios, la ansiedad en diecinueve y ambas medidas en catorce.

En el meta-análisis de ansiedad se incluyó una muestra total de mil veintitrés personas; se determinó que el tamaño del efecto combinado de las intervenciones fue moderado y 
robusto. Por su parte, el tamaño del efecto combinado en el meta-análisis de depresión, que incluyó una muestra de mil ciento un personas, fue robusto, pero menor que el observado en el de ansiedad. En cuanto al tipo de intervención, determinaron que la terapia grupal fue tan efectiva como la terapia individual, y que las intervenciones intensivas pero cortas llevadas a cabo por terapeutas con más experiencia produjeron mejores resultados.

De forma similar, con el objetivo de analizar el efecto general de diversos tratamientos psicológicos en la calidad de vida de pacientes y sobrevivientes de cáncer, De la TorreLuque, Gambara, López y Cruzado (2016) llevaron a cabo un meta-análisis incluyendo artículos con una muestra de personas adultas con cáncer y sobrevivientes de cáncer, que analizaran el efecto de intervenciones psicológicas (entrenamiento en habilidades de afrontamiento, psicoeducación, relajación y psicoterapia) en la calidad de vida, y que incluyeran un grupo control. Un total de setenta y ocho estudios fueron incorporados con un total de siete mil trescientos cuarenta y dos participantes, y con un diagnóstico de cáncer de mama en un $46.20 \%$ de los casos.

Se determinó que las intervenciones tuvieron un tamaño del efecto estadísticamente significativo de pequeño a mediano en la calidad de vida de las personas participantes, no encontrándose efectos de otros moderadores, incluyendo factores médicos y aspectos relacionados con las intervenciones. Además, se encontró que los tratamientos psicológicos que reducían síntomas depresivos promovieron mejores niveles de calidad de vida en pacientes con cáncer.

En una línea similar, Yang et al. (2014) realizaron un meta-análisis con el objetivo de determinar la efectividad de intervenciones psicológicas en general en el tratamiento de la depresión y la ansiedad en pacientes adultos con cáncer, tomando en cuenta los resultados de ensayos controlados aleatorizados. Entre los tratamientos incluidos, se encontraron intervenciones cognitivo-conductuales, educación, relajación, apoyo familiar y social, terapia musical e intervenciones desde la enfermería. Se incluyeron ciento cuarenta y tres estudios con un total de catorce mil treinta y nueve sujetos de estudio.

Así, se determinaron tamaños del efecto grandes, tanto para depresión como para ansiedad, aunque se encontraron altos niveles de heterogeneidad entre estudios. Asimismo, se obtuvieron efectos significativos según las variables moderadoras de tipo de cáncer (con efectos más grandes en cáncer de pulmón), la selección de pacientes (la preselección de pacientes con signos claros de ansiedad y depresión mostró efectos mayores), cuestionarios utilizados (con tamaños del efecto más grandes en la investigaciones que utilizaron el instrumento Inventario de Ansiedad Estado-Rasgo), y formato de la intervención (la psicoterapia individual mostró tamaños de efecto más grandes).

Como se observa, tanto las intervenciones aplicadas como los resultados obtenidos son diversos. Precisamente, varias investigaciones de las presentadas resaltan la necesidad de un diseño y aplicación de estudios rigurosos. De igual manera, se desprende de la revisión efectuada que no se han llevado a cabo suficientes estudios meta-analíticos específicos en relación con la efectividad de las terapias psicológicas sobre la ansiedad en personas con cáncer de mama. Por tanto, resulta de importancia una aproximación al tema que permita determinar la efectividad de los tratamientos utilizados con esta población. 


\section{Método}

La investigación se enfocó en la elaboración de un meta-análisis que permitiera un análisis comprehensivo de los datos obtenidos en investigaciones publicadas sobre el tema de interés. Como plantean Botella y Gambara (2002), el meta-análisis permite, a través de procedimientos cuantitativos, la integración y el análisis de resultados de investigaciones científicas enfocadas en un área específica. De esta manera, se buscó determinar el tamaño de efecto global de las intervenciones psicológicas sobre la ansiedad en personas con cáncer de mama.

Las fases de estudio fueron las usuales para el desarrollo de un meta-análisis: formulación del problema de estudio, definición de los criterios de inclusión, búsqueda de literatura que permita el análisis, creación de la base de datos y codificación de los resultados, aplicación de análisis estadísticos e interpretación de los resultados, y discusión de los hallazgos a través del contraste con la literatura relacionada con el tema (Botella \& Gambara, 2002; Marín, Sánchez, \& López, 2009).

Se plantearon los siguientes criterios de inclusión previos a la búsqueda de artículos: investigaciones cuya población fuera de mujeres adultas; estudios revisados por pares (peer-reviewed); publicaciones desde el año 1994, año en que se publicó la cuarta versión del Manual Diagnóstico y Estadístico de los Trastornos Mentales por parte de la Asociación Estadounidense de Psicología, donde se refinaron y modificaron los criterios para el diagnóstico de los trastornos de ansiedad; investigaciones de tipo experimental y cuasiexperimental; investigaciones que midieran el nivel de ansiedad con el uso de instrumentos de calidad; estudios que trabajaran con población con cáncer de mama; publicaciones en español o en inglés; estudios que contaran con un grupo control; investigaciones que incluyeran en sus resultados los datos estadísticos necesarios que permitieran el cálculo del tamaño del efecto, es decir, la media y la desviación estándar pretest y postest del grupo experimental y del grupo control.

Al tratarse de una investigación meta-analítica, la información para el análisis se obtuvo de artículos publicados de experimentos y cuasi-experimentos. De manera que se realizó una revisión sistemática entre octubre de 2016 y agosto de 2017 de los artículos publicados sobre el tema de estudio. Como explican Littell, Corcoran y Pillai (2008), una revisión sistemática tiene como objetivo localizar y sintetizar, de forma comprehensiva y disciplinada, la investigación existente en torno a un tema específico; utiliza procedimientos organizados, transparentes y replicables en todas las etapas del proceso, a la vez que busca minimizar el error y el sesgo.

Los descriptores o palabras clave en español que se utilizaron en esta búsqueda fueron: terapia o intervención o tratamiento $\mathrm{Y}$ psicológico o psicoterapia o psico oncológico Y ansiedad Y cáncer o cáncer de mama o carcinoma u oncológico. Por su parte, en inglés se utilizaron las siguientes: psychological or psychotherapy or psycho oncological AND intervention or therapy or treatment AND anxiety AND cancer or breast cancer or carcinoma or oncological. Así, de los 49129 resultados iniciales, 17392 se obtuvieron con la cadena de palabras en español y 31737 con su equivalente en inglés.

Las bases de datos en las que se llevó a cabo la búsqueda fueron Dialnet, Ebsco, Latindex, PubMed, ScienceDirect, Wiley, SAGE, Scielo y Google Scholar. Estas bases se eligieron 
con el fin de obtener acceso a una gran variedad de publicaciones relacionadas con el tema de interés, tanto en español como en inglés. Con base en los criterios de elegibilidad, se descartaron los resultados que no cumplían con los requisitos planteados, y se efectuó la selección de artículos con los que se llevó a cabo el meta-análisis.

De los artículos que cumplieron los criterios tras la primera revisión por título, se procedió a la lectura de los resúmenes o abstracts, con el fin de determinar cuáles continuaban siendo pertinentes y cuáles no cumplían con los requisitos necesarios para su inclusión en el metaanálisis. Posteriormente, se procedió a la lectura de los artículos completos elegidos tras la lectura de los resúmenes, con el objetivo de realizar la selección final de investigaciones que formaron parte del meta-análisis.

Se incluyeron los datos de medias y desviaciones estándar, tanto de los grupos experimentales como de los grupos control o de comparación, lo que permitió el cálculo del tamaño del efecto global. Además, se planteó, realizar un análisis de variables moderadoras, el cual, como manifiestan Littell et al. (2008), permite explorar potenciales moderadores del efecto del tratamiento, con el fin de determinar en qué circunstancias los tratamientos son más efectivos y qué variables tienen influencia en el tamaño de efecto global. Debido a la variabilidad entre estudios y a la cantidad de artículos considerados, no fue posible llevar a cabo el análisis de variables moderadoras categóricas, el cual sí se realizó con las variables continuas.

Para la evaluación de la calidad de los estudios se utilizó como base la escala Jadad (Jadad et al., 1996), con un ítem adicional relacionado con la presencia o ausencia de seguimiento. Se tomaron como criterios de calidad la presencia de aleatorización, doble ciego, explicación del manejo de la muerte experimental y seguimiento, otorgando un punto por cada criterio presente, con una puntuación máxima de cuatro y una mínima de cero.

La información extraída de los artículos seleccionados se analizó a través del programa estadístico Comprehensive Meta-Analysis (Biostat, Inc., versión 3.0). De esta forma, el estadístico utilizado para el tamaño del efecto fue la $g$ de Hedges, el cual, como plantean Sheard y Maguire (1999), permite obtener esta información estimando una diferencia media estandarizada, que se obtiene como la media del grupo experimental menos la media del grupo control dividido entre la desviación estándar conjunta. La $g$ de Hedges es muy utilizada en los estudios meta-analíticos, ya que puede utilizarse con cualquier tamaño de muestra, pero es especialmente útil cuando muestras pequeñas se incluyen, pues permite compensar el sesgo por muestra pequeña (Littell et al., 2008). Así, es una corrección comúnmente utilizada de la fórmula original de la diferencia de medias estandarizada.

Para el tamaño del efecto, un signo positivo hace referencia a un aumento, mientras un signo negativo hace referencia a una disminución. De esta forma, el signo esperado en este meta-análisis era uno negativo, pues todos los instrumentos de medición reflejaban el nivel de ansiedad; entonces, lo esperado era que las intervenciones redujeran estas puntuaciones. Como explica Faraone (2008), si una mejoría se relaciona con puntuaciones más bajas en el instrumento de medición (como es este caso, ya que los instrumentos miden el nivel de ansiedad), una diferencia de medias estandarizada menor que cero (con signo negativo) indica el nivel en el que el tratamiento es más eficaz que el placebo. 
La significancia del tamaño de efecto fue obtenida a través de un intervalo de confianza de un $95 \%$ y la prueba $Z$.

Asimismo, se realizó una prueba de homogeneidad $Q$, que permite conocer si los tamaños de efecto individuales son homogéneos y representan una medida similar de la eficacia del tratamiento (Thomas \& Nelson, 2007). De igual manera, se hizo la prueba $I^{2}$, la cual indica el porcentaje en que los tamaños de efecto individuales son heterogéneos, de forma que un valor de $25 \%$ sería bajo, uno de $50 \%$ sería medio y uno de $75 \%$ correspondería a un nivel alto de heterogeneidad (Borenstein, Hedges, Higgins, \& Rothstein, 2009).

Por su parte, se utilizó un modelo aleatorio para los análisis estadísticos, el cual se basa en el supuesto de que el efecto puede cambiar entre estudios y muestras debido a diversas variables. De esta manera, asume que las investigaciones incluidas son una muestra aleatoria de la distribución relevante de los efectos y que el efecto principal se ve influenciado por variaciones en los estudios, además de errores de muestreo dentro de estos, por lo que toma en consideración una mayor cantidad de fuentes de variación (Littell et al., 2008).

Como plantean Ahmed, Sutton y Riley (2012), el sesgo de publicación se presenta cuando los estudios con resultados positivos, estadística o clínicamente, tienen más probabilidad de ser publicados. En este sentido, el cálculo del sesgo busca estimar la cantidad de tamaños de efecto no significativos que son requeridos para disminuir el tamaño de efecto global a uno pequeño o no significativo (Thomas \& Nelson, 2007). Para el cálculo de este sesgo se utilizó la ecuación de Hedges y Olkin (1985); la fórmula se presenta en la Ecuación 1.

$$
K_{o}=\frac{K\left(d_{1}-d_{2}\right)}{d_{2}}
$$

De esta forma, $K_{0}$ hace referencia al número de estudios requeridos para reducir el tamaño de efecto global a uno pequeño o no significativo, $K$ es el número total de tamaños de efecto incluidos en el meta-análisis, $d_{1}$ es la media de todos los tamaños de efecto del metaanálisis y $d_{2}$ hace referencia a un tamaño de efecto no significativo (en este caso, se utilizó el valor de $\left.d_{2}=-.20\right)$. En línea con los análisis anteriores, se utilizó un funnel plot y un análisis de regresión de Egger, con el propósito de medir el sesgo en general.

\section{Resultados}

Tras la revisión de artículos realizada en las bases de datos, diecisiete investigaciones cumplieron los criterios necesarios para la inclusión en el estudio. En la Figura 1 se presenta el proceso de selección de artículos de acuerdo con las etapas seguidas.

Un total de veinte tamaños de efecto fueron incluidos, debido a que dos estudios (Dolbeault et al., 2009; Nunes et al., 2007) reportaron resultados desprendidos de dos instrumentos independientes para la medición de la ansiedad, y el estudio de Sarenmalm, Mårtensson, Andersson, Karlsson y Bergh (2017) utilizó dos grupos de comparación, uno activo y 
uno pasivo. En la Tabla 1 se presentan las medias y desviaciones estándar reportadas en las investigaciones realizadas, con base en las cuales se calculó el tamaño del efecto. De acuerdo con los criterios para la evaluación de la calidad seleccionados, los estudios incluidos tuvieron puntuaciones entre uno y cuatro $(M=3.00, D T=1.08)$, de un rango posible entre cero y cuatro. La totalidad de las participantes $(N=2283)$ fueron mujeres. Las participantes asignadas a la condición experimental en los estudios fueron, en total, 1126 mujeres, mientras que las asignadas a la condición de comparación fueron 1157.

Resultados obtenidos en la búsqueda

en bases de datos $(n=49129)$

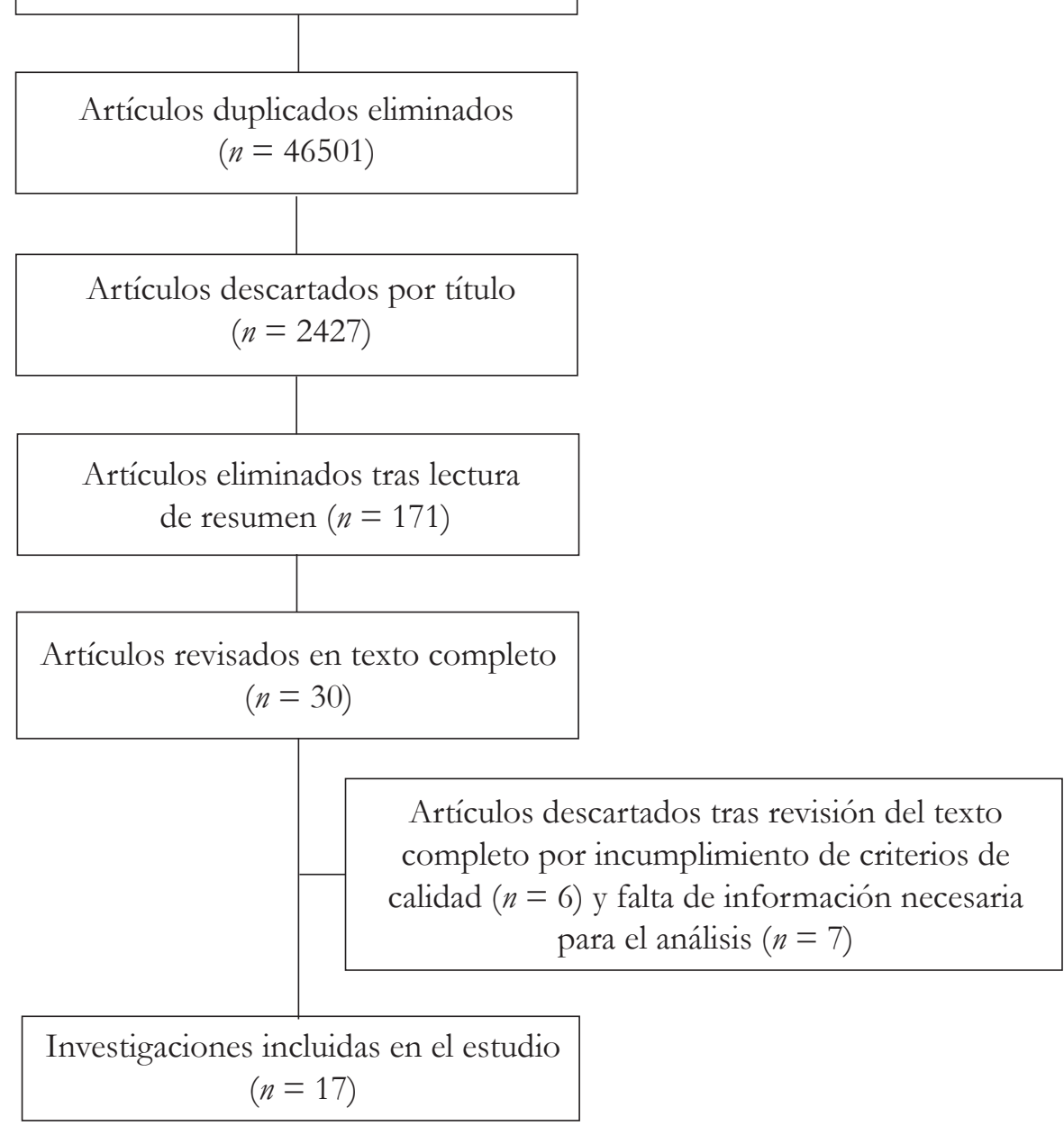

Figura 1. Proceso de selección de artículos 


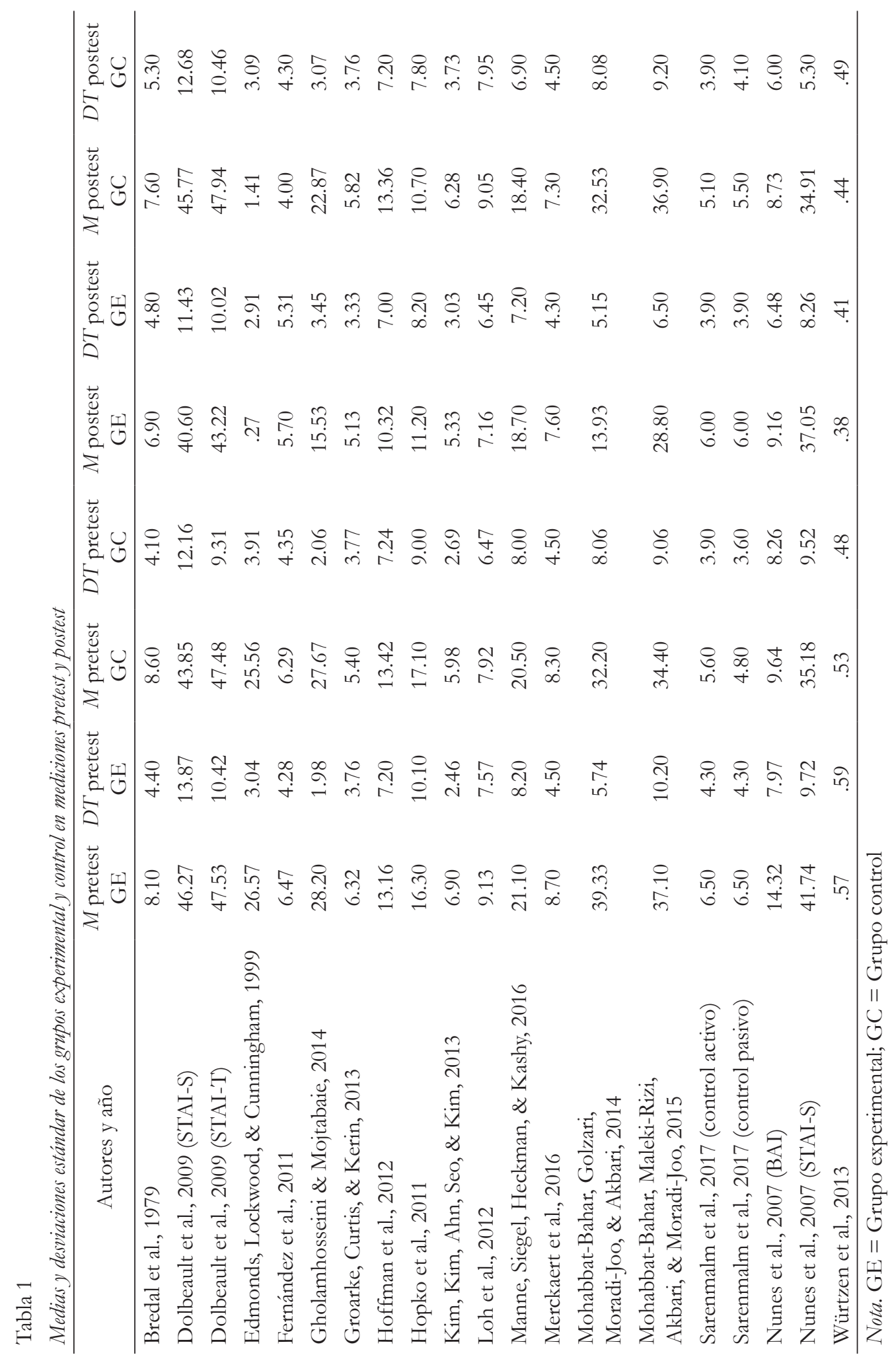

Actualidades en Psicología, 34(128), 2020, 121-141 
La mayor parte de los estudios fueron de tipo experimental $(n=13)$, mientras que hubo gran variabilidad en los países en los que se desarrollaron las investigaciones incluidas. De esta manera, tres investigaciones se llevaron a cabo en Irán, dos en Estados Unidos de América, y los demás artículos incluidos presentaron estudios realizados en países de Europa principalmente (Irlanda, Francia, España, Bélgica, Suecia, Dinamarca, Inglaterra y Noruega), así como países de Asia (Malasia) y América (Brasil).

En relación con la edad de las participantes, las medias se encontraron en un rango entre 47.49 y 57.20 años. Todas las participantes habían sido diagnosticadas con cáncer de mama y habían recibido o se encontraban en proceso de recibir tratamiento médico (radioterapia, quimioterapia o intervención quirúrgica). En cuanto a los años de publicación, los estudios incorporados se publicaron entre el año 1999 y el año 2017.

Por su parte, sobre los tipos de intervenciones utilizadas en las investigaciones, la mayor parte se concentró en las categorías de abordaje cognitivo-conductual $(n=7)$ y terapias de tercera generación $(n=7)$, aunque se incluyeron dos artículos con abordaje humanista y uno con intervención psicoanalítica. En cuanto a la estructura de las intervenciones, el número de sesiones se encontró entre cuatro y treinta y seis, a lo largo de entre tres y media y treinta y cinco semanas de abordaje. De esta manera, la duración total de las intervenciones se mantuvo entre cuatro y media y ochenta y cuatro horas. Finalmente, la mayor parte de las intervenciones fue de tipo grupal $(n=15)$. En la Tabla 2 se presenta el tipo de intervención psicológica específico de cada artículo considerado en la investigación, así como los instrumentos utilizados en cada uno para la medición de la ansiedad.

En cuanto a las condiciones de comparación, doce estudios utilizaron condiciones pasivas (sin ningún tipo de intervención terapéutica o educativa), mientras que cuatro estudios emplearon condiciones activas (entre las que se incluía la difusión de material educativo, el entrenamiento en habilidades y la formación de grupos de apoyo) y un estudio (Sarenmalm et al., 2017) utilizó ambas. En la Tabla 3 se presentan los tamaños de efecto individuales en cada uno de los estudios analizados.

El tamaño de efecto global obtenido fue de $g=-.459(n=20$, IC 95\% $=-.280-.637$, $\left.Z=-5.024, p<.001, Q=86.785, d f=19, \mathrm{I}^{2}=78.107, p<.001\right)$. De acuerdo con los lineamientos propuestos por Cohen (citado en Littell et al., 2008), el tamaño del efecto obtenido se ubicaría entre un tamaño de efecto pequeño (.2) y uno mediano (.5); por su parte el análisis de $I^{2}$ mostró que hay un nivel elevado de heterogeneidad en los tamaños de efecto individuales. En cuanto al análisis de sesgo, se determinó un valor de $K_{0}=26$, que utiliza un valor de tamaño de efecto no significativo de -.200. En la Figura 2, se muestra el forest plot, o diagrama de efectos, correspondiente a los resultados obtenidos.

En relación con el análisis de variables moderadoras categóricas que se planteó efectuar al inicio de la investigación, la variabilidad entre las características de los estudios y las restricciones estadísticas para este tipo de análisis con la cantidad de investigaciones encontradas no lo permitió. Sin embargo, se realizaron análisis de meta-regresión con las variables moderadoras continuas. En la Tabla 4 se presenta el detalle de los análisis de meta-regresión según la variable continua estudiada. 
Tabla 2

Tipo de intervención psicológica e instrumento de medición de ansiedad según estudio

\begin{tabular}{|c|c|c|c|}
\hline Autores y año & Técnica de intervención & Modalidad & Instrumento de medición \\
\hline Bredal et al., 1979 & Psicoeducación & Grupal & $\begin{array}{l}\text { Hospital Anxiety and Depression } \\
\text { Scale (HADS) }\end{array}$ \\
\hline \multirow{2}{*}{$\begin{array}{l}\text { Dolbeault et } \\
\text { al., } 2009\end{array}$} & \multirow[t]{2}{*}{ Psicoeducación } & \multirow[t]{2}{*}{ Grupal } & Profile of Mood States (POMS) \\
\hline & & & $\begin{array}{l}\text { State-Trait Anxiety Inventory } \\
\text { (STAI) }\end{array}$ \\
\hline Edmonds et al., 1999 & $\begin{array}{l}\text { Intervención } \\
\text { psicológica prolongada } \\
\text { (apoyo, solución de } \\
\text { problemas, manejo } \\
\text { de emociones) }\end{array}$ & Grupal & $\begin{array}{l}\text { Mental Adjustment to Cancer } \\
\text { Scale (MAC) }\end{array}$ \\
\hline $\begin{array}{l}\text { Fernández et } \\
\text { al., } 2011\end{array}$ & Activación conductual & Individual & $\begin{array}{l}\text { Hospital Anxiety and Depression } \\
\text { Scale (HADS) }\end{array}$ \\
\hline $\begin{array}{l}\text { Gholamhosseini \& } \\
\text { Mojtabaie, } 2014\end{array}$ & $\begin{array}{l}\text { Terapia de aceptación } \\
\text { y compromiso }\end{array}$ & Grupal & Beck Anxiety Inventory (BAI) \\
\hline Groarke et al., 2013 & $\begin{array}{l}\text { Manejo de estrés desde } \\
\text { terapia cognitivo- } \\
\text { conductual }\end{array}$ & Grupal & $\begin{array}{l}\text { Hospital Anxiety and Depression } \\
\text { Scale (HADS) }\end{array}$ \\
\hline Hoffman et al., 2012 & Mindfulness & Grupal & Profile of Mood States (POMS) \\
\hline Hopko et al., 2011 & Solución de problemas & Individual & Beck Anxiety Inventory (BAI) \\
\hline Kim et al., 2013 & Meditación guiada & Grupal & $\begin{array}{l}\text { Hospital Anxiety and Depression } \\
\text { Scale (HADS) }\end{array}$ \\
\hline Loh et al., 2012 & $\begin{array}{l}\text { Auto-manejo y } \\
\text { manejo emocional }\end{array}$ & Grupal & $\begin{array}{l}\text { Depression, Anxiety and Stress } \\
\text { tool (DASS) }\end{array}$ \\
\hline Manne et al., 2016 & Intervención de pareja & Grupal & Mental Health Inventory-38 \\
\hline $\begin{array}{l}\text { Merckaert et } \\
\text { al., } 2016\end{array}$ & $\begin{array}{l}\text { Terapia cognitivo- } \\
\text { conductual e hipnosis }\end{array}$ & Grupal & $\begin{array}{l}\text { Hospital Anxiety and Depression } \\
\text { Scale (HADS) }\end{array}$ \\
\hline $\begin{array}{l}\text { Mohabbat-Bahar } \\
\text { et al., } 2014\end{array}$ & Logoterapia & Grupal & Beck Anxiety Inventory (BAI) \\
\hline $\begin{array}{l}\text { Mohabbat-Bahar } \\
\text { et al., } 2015\end{array}$ & $\begin{array}{l}\text { Terapia de aceptación } \\
\text { y compromiso }\end{array}$ & Grupal & Beck Anxiety Inventory (BAI) \\
\hline $\begin{array}{l}\text { Sarenmalm et } \\
\text { al., } 2017\end{array}$ & Mindfulness & Grupal & $\begin{array}{l}\text { Hospital Anxiety and Depression } \\
\text { Scale (HADS) }\end{array}$ \\
\hline \multirow[t]{2}{*}{ Nunes et al., 2007} & \multirow{2}{*}{$\begin{array}{l}\text { Relajación y } \\
\text { visualización }\end{array}$} & \multirow[t]{2}{*}{ Grupal } & Beck Anxiety Inventory (BAI) \\
\hline & & & $\begin{array}{l}\text { State-Trait Anxiety Inventory } \\
\text { (STAI) }\end{array}$ \\
\hline Würtzen et al., 2013 & Mindfulness & Grupal & $\begin{array}{l}\text { Symptom Checklist-90-r (SCL- } \\
90 \mathrm{r})\end{array}$ \\
\hline
\end{tabular}




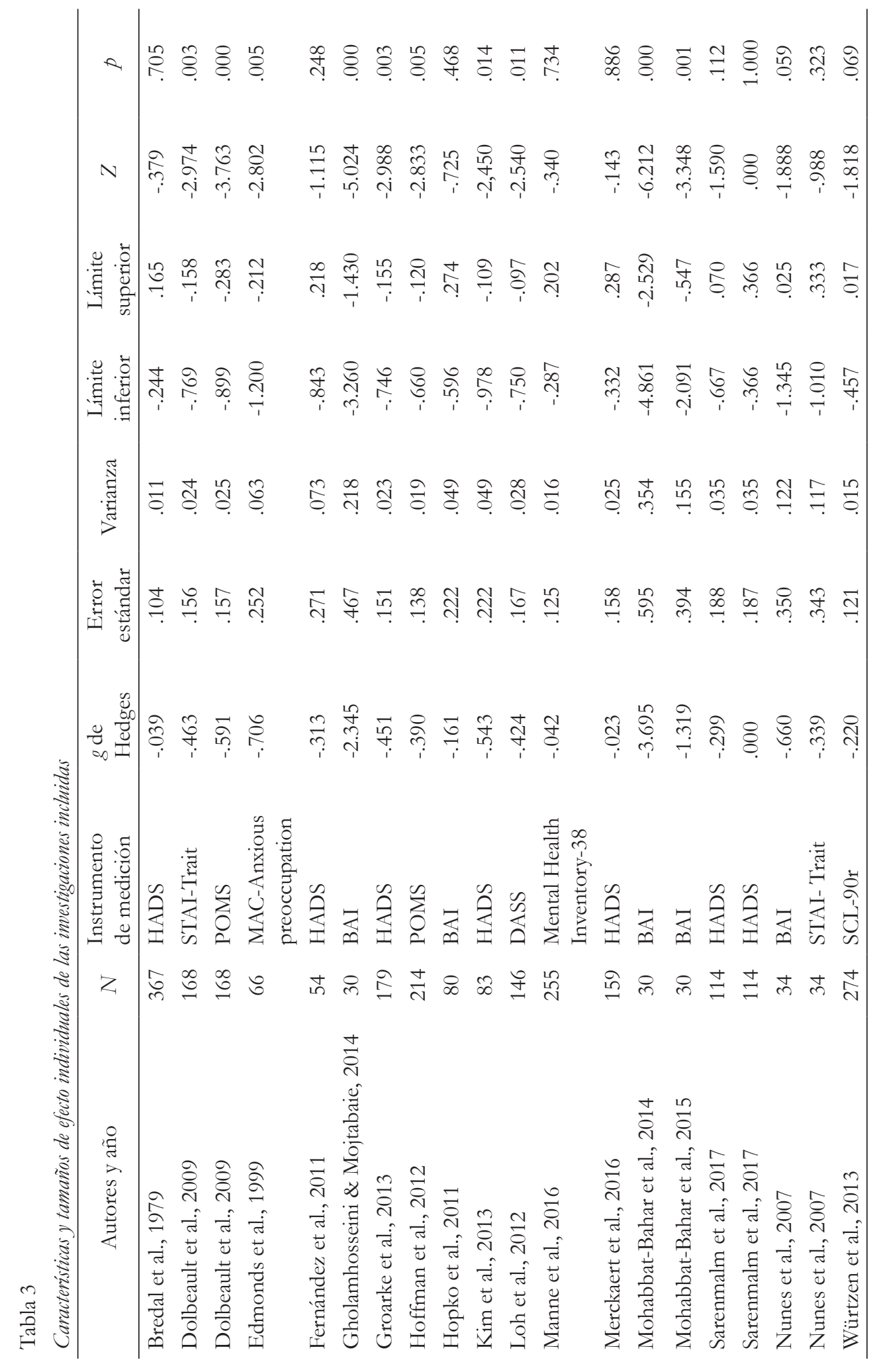

Actualidades en Psicología, 34(128), 2020, 121-141 
Bredal et al. (1979)

Dolbeault et al. (2009)

Dolbeault et al. (2009)

Edmonds et al. (1999)

Fernández et al. (2011)

Gholamhosseini \& Mojtabaie (2014)

Groarke et al. (2013)

Hoffman et al. (2012)

Hopko et al. (2011)

Kim et al. (2013)

Loh et al. (2012)

Manne et al. (2016)

Merckaert et al. (2016)

Mohabbat-Bahar et al. (2014)

Mohabbat-Bahar et al. (2015)

Sarenmalm et al. (2017)

Sarenmalm et al. (2017)

Telles Nunes et al. (2007)

Telles Nunes et al. (2007)

Würtzen et al. (2013)
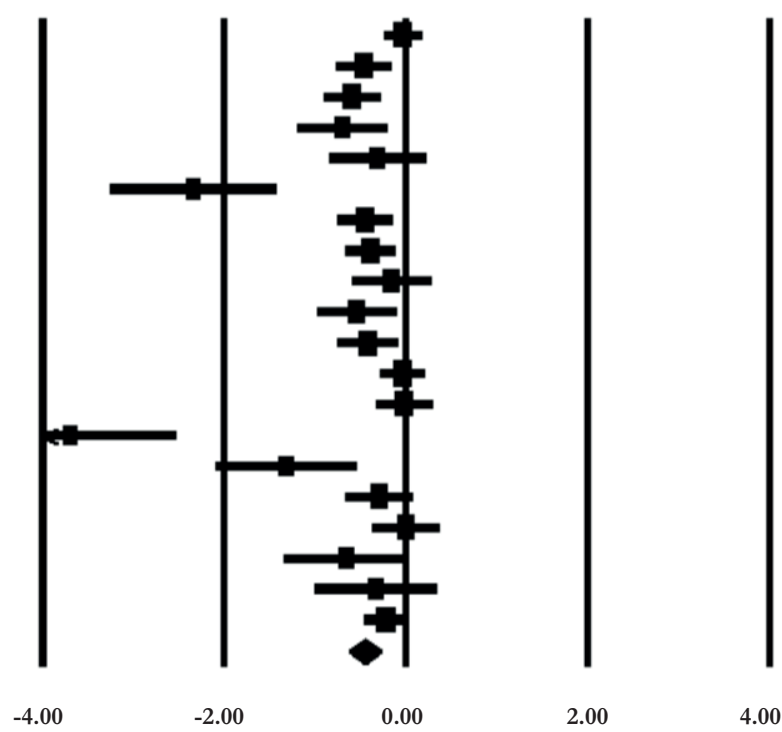

Figura 2. Diagrama de efectos de los estudios incorporados.

Tabla 4

Análisis de meta-regresión de variables continuas

\begin{tabular}{lccc}
\hline \multicolumn{1}{c}{ Variable continua } & $\mathrm{Q}$ & $\mathrm{Df}$ & $p$ \\
\hline Año & 12.159 & 1.000 & $<.001$ \\
Cantidad de sesiones & 1.986 & 1.000 & .159 \\
Horas totales de intervención & .902 & 1.000 & .342 \\
Semanas totales de intervención & .100 & 1.000 & .752 \\
Calidad & 4.734 & 1.000 & .029 \\
Edad & 10.192 & 1.000 & .001 \\
\hline
\end{tabular}

De acuerdo con estos resultados, el año, la edad y la calidad de los estudios presentaron efectos significativos sobre la efectividad de las intervenciones. De esta manera, a mayor edad de las participantes y en estudios con una mayor puntuación en la escala de calidad utilizada, se obtendría un mayor tamaño del efecto de las intervenciones. De forma similar, estudios realizados en años posteriores parecen obtener reducciones más grandes en el nivel de ansiedad. Por su parte, las variables de cantidad de sesiones, horas de intervención y semanas totales de intervención no presentaron resultados significativos.

En cuanto al análisis de sesgo en relación con estudios no publicados, en la Figura 3 se presenta el funnel plot de errores estándar según la $g$ de Hedges. En relación con esto, para el análisis de regresión de Egger, se obtuvo un valor de $t=4.604$ ( $d f=18, p<.001)$. De esta manera, si el valor del análisis de regresión se aleja de cero, hay una asimetría más 


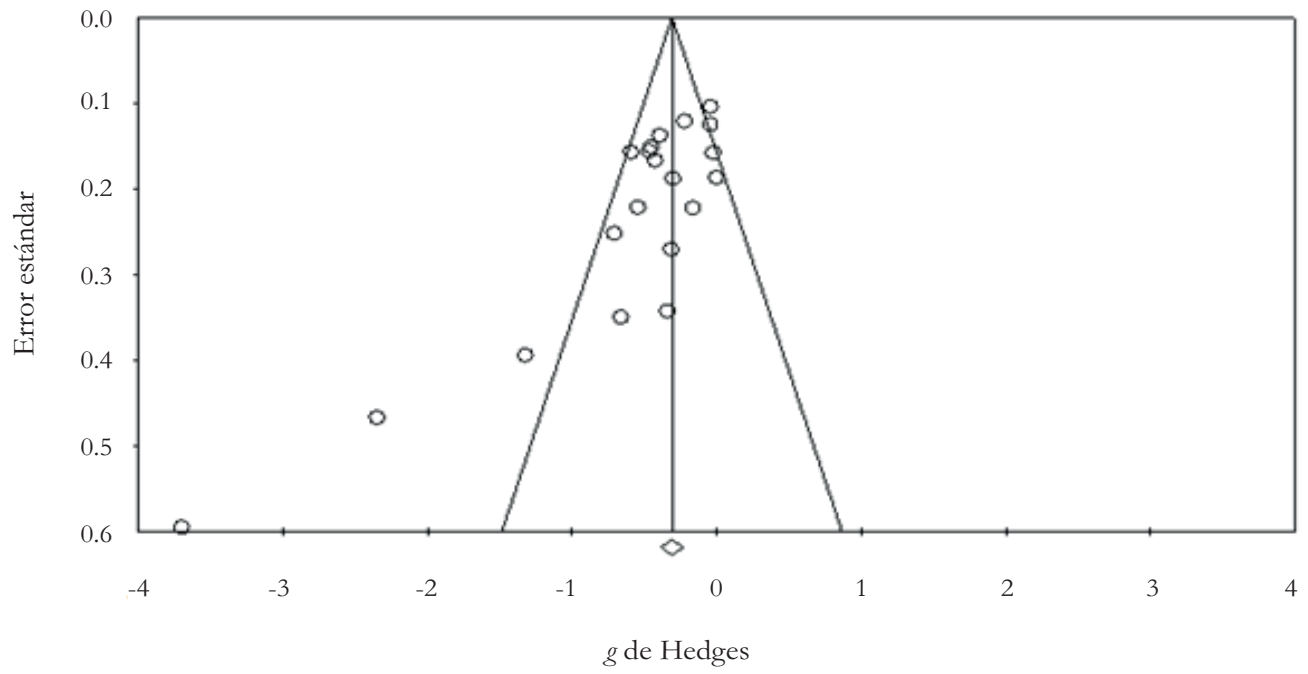

Figura 3. Funnel plot de errores estándar según $g$ de Hedges.

pronunciada, la cual, en este caso, es estadísticamente significativa. Como plantean Littell et al. (2008), esta asimetría puede deberse a la presencia de sesgo de publicación, aunque también puede relacionarse con diseños y métodos analíticos poco rigurosos en estudios con muestras pequeñas.

\section{Discusión}

El objetivo general de la investigación consistió en la estimación del tamaño de efecto global de las intervenciones psicológicas para el tratamiento de la ansiedad en personas con cáncer de mama. De esta manera, se consiguió el objetivo al determinar que las intervenciones al respecto tuvieron un tamaño del efecto global de $g=-.459$, el cual se consideraría un tamaño de pequeño a mediano. El elemento de importancia que brinda esta investigación, en este sentido, es el de proporcionar datos generales que guíen la práctica clínica en estos casos, tomando en cuenta si los efectos de los tratamientos sobre el nivel de ansiedad son apreciables.

Los resultados obtenidos se corresponden con los encontrados en otros estudios similares. En el meta-análisis realizado por Tao, Jiang, Liu, Aungsuroch y Tao (2015), se estudió el tamaño del efecto de intervenciones psico-oncológicas sobre el sufrimiento o distress en pacientes de ambos sexos con cáncer; el equipo de investigación encontró tamaños de efecto medianos (comprendidos entre $d=.53$ y $d=.56$ ). De forma similar, Sheard y Maguire (1999) realizaron un meta-análisis para estudiar los efectos de distintas psicoterapias en los niveles de ansiedad y depresión en personas con cáncer. El tamaño de efecto general fue de $d=.42$, similar al encontrado en este meta-análisis.

En relación con técnicas específicas, Cramer, Lauche, Paul y Dobos (2012) tuvieron como objetivo estudiar la efectividad del mindfulness sobre los niveles de depresión y ansiedad en pacientes con cáncer; el tamaño del efecto global encontrado fue bajo, en comparación 
con el tratamiento usual. Sin embargo, cabe mencionar que ninguno de los estudios trabajó con personas con cáncer de mama, aunque los investigadores se habían planteado incluir a esta población.

Como puede observarse en los meta-análisis mencionados, se obtuvieron resultados favorables, de pequeños a medianos, en investigaciones similares a esta investigación meta-analítica, sin encontrarse en esta búsqueda meta-análisis que concluyan que las intervenciones psicológicas no obtienen resultados terapéuticos estadísticamente significativos u obtienen resultados desfavorables en las variables dependientes de estudio. A pesar de que las características entre los estudios presentan variabilidad, puede observarse que las intervenciones psicoterapéuticas, de forma general, parecen favorecer la reducción de los niveles de ansiedad en personas con un diagnóstico de cáncer, y pueden brindar herramientas útiles para hacer frente a los retos que presenta ese diagnóstico.

Sin embargo, cabe la posibilidad de preguntarse si estos resultados justifican la inversión de recursos (temporales, económicos, institucionales, profesionales, entre otros) que forman parte de intervenciones de este tipo. La necesidad de involucramiento de profesionales en psicología es evidente, al tomar en cuenta la presencia elevada de diagnósticos relacionados con la salud mental que se realizan en esta población. Aun así, es de importancia cuestionarse si las intervenciones están siendo suficientemente eficaces de la manera en que se están implementando actualmente o si es posible replantearse las características de los tratamientos, de la preparación de profesionales y de la forma general en que se llevan a cabo.

En relación con el análisis de variables continuas, se determinó que únicamente tres de las estudiadas (el año, la edad y la calidad de los estudios) obtuvieron efectos significativos sobre el tamaño del efecto de las intervenciones. De esta manera, a mayor edad, en estudios de mayor calidad y en investigaciones efectuadas en años posteriores, se obtendría una mayor reducción en los niveles de ansiedad. Sin embargo, es necesario tomar ciertas precauciones en la lectura de estos resultados, en relación con la causa de estas conexiones observadas.

Así, es posible que estudios más recientes y de mayor calidad utilicen criterios y métodos más rigurosos que favorezcan resultados positivos. De igual forma, es posible que personas de mayor edad cuenten con distintas herramientas que les permitan obtener mayores beneficios de las intervenciones. Sin embargo, al tratarse de hipótesis y no de relaciones probadas, es necesario que estudios posteriores busquen esclarecer si efectivamente existe una relación entre estas variables y el tamaño del efecto y, de ser así, qué elementos se ven involucrados, con el objetivo de comprender y utilizar esta información para el beneficio de las personas participantes.

En esta investigación meta-analítica, tres artículos sobresalieron en el diagrama de efectos por sus tamaños de efecto elevados (Gholamhosseini \& Mojtabaie, 2014; MohabbatBahar et al., 2014; Mohabbat-Bahar et al., 2015); sus resultados pueden relacionarse, posiblemente, con elementos del diseño metodológico o del análisis de datos. Cabe mencionar que, al excluir estos tres artículos, el tamaño de efecto obtenido es de $g=-.295$ $(\phi<.001)$; se observa que el tamaño del efecto disminuye, pero se mantiene en el rango de pequeño a mediano, y continúa siendo significativo. 
Sobre las limitaciones de este estudio, cabe resaltar la imposibilidad de realizar el análisis de variables moderadoras categóricas, lo cual permitiría obtener información sobre cuáles tipos de intervención resultan más efectivos. En este sentido, diferencias marcadas entre los estudios encontrados y falta de investigaciones que representaran ciertas posibilidades de intervención no permitieron el estudio de los efectos de estas variables sobre los resultados obtenidos.

De esta forma, la baja cantidad de investigación disponible desde intervenciones diferentes a los tratamientos cognitivo-conductuales y de tercera generación es un elemento para tomar en consideración. Debido a que no es posible determinar la eficacia de este tipo de intervenciones sin medidas adecuadas y controladas de sus resultados, no se puede justificar con evidencia su aplicación en pacientes con este tipo de diagnósticos. En este sentido, la responsabilidad de brindarle la mejor alternativa de atención posible a las pacientes debería motivar un mayor desarrollo de estudios en las terapias que se pretendan aplicar, cualquiera que sea su base teórica.

Otra limitación de esta investigación es la inclusión de investigaciones cuasi-experimentales, debido a que en diversos meta-análisis estos son excluidos por considerarse de menor calidad. Sin embargo, la inclusión de una escala de calidad para los estudios permitió tomar en cuenta esta diferencia e incluirla en el análisis. De igual manera, la mayor parte de estudios considerados fueron de diseño experimental.

Por su parte, la inclusión únicamente de artículos publicados es una limitación de esta investigación. Como plantean Tao et al. (2015), existe un sesgo en cuanto a lo que se publica y se reporta, que suele favorecer estudios con resultados más positivos. De esta manera, el sesgo de publicación hace referencia a la posibilidad de que las investigaciones publicadas no representen la totalidad de estudios de alta calidad en un tema (Littell et al., 2008).

A partir de estas limitaciones y de los resultados obtenidos, ciertas recomendaciones pueden realizarse. Para investigaciones aplicadas, es necesario el uso de metodologías de calidad, que permitan aportar en sí mismas y en estudios de mayor escala (revisiones sistemáticas y meta-análisis), teniendo como prioridad la rigurosidad y la calidad de la información reportada, sea esta o no favorable a las hipótesis de investigación. La aleatorización a las condiciones experimental y de comparación, y el reporte de las mediciones pretest y postest, resultan de gran importancia para mejorar la calidad de los estudios y la posibilidad de análisis.

Asimismo, el elemento de la replicabilidad tiene un papel de gran importancia en el desarrollo del saber basado en evidencia. Como explican Nelson, Simmons y Simonsohn (2018), por mucho tiempo la psicología experimental se apoyó en métodos de obtención y análisis de datos que hacían sencilla la publicación de falsos positivos, lo cual imposibilita la distinción entre resultados replicables y resultados falsos y no replicables. Así, de manera reciente se ha dado un cambio hacia una introspección metodológica, llegando a la conclusión de que muchos estudios han sido tratados de forma que las publicaciones son selectivas, pues manejan los datos a conveniencia de los objetivos e hipótesis de investigación (Nelson et al., 2018). 
En este sentido, se ha recomendado un reporte menos selectivo de los métodos, análisis y resultados, al evidenciar las medidas utilizadas, las manipulaciones y exclusiones de información, así como el preregistro de los planes de análisis de datos antes de llevarlos a cabo (Nelson et al., 2018). Asimismo, como plantean Nelson et al. (2018), la replicación de estudios realizados es un paso hacia el aumento de la integridad y credibilidad de la disciplina psicológica, con el propósito de fortalecer y de afinar la manera en que, desde ella, se recaba, analiza y presenta la información que guía la práctica.

Por su parte, como se mencionó anteriormente, desarrollar más investigaciones y publicaciones de calidad desde distintos enfoques de intervención ampliamente utilizados en la práctica clínica con esta población, como lo son las intervenciones humanistas, psicoanalíticas y de otras corrientes, resultaría de gran importancia, con el fin de conocer si están teniendo resultados positivos para las personas participantes. De igual manera, el uso de comparaciones activas es de interés a fin de conocer cuáles técnicas pueden presentar beneficios específicos sobre otras.

Asimismo, es de gran relevancia que este tipo de investigaciones se lleven a cabo en contextos latinoamericanos, tomando en cuenta la cantidad de diagnósticos de cáncer en general, y cáncer de mama específicamente, que se realizan en esta población. Es necesario conocer si la salud mental de las personas que reciben este diagnóstico está siendo adecuadamente atendida, de qué forma, y en qué medida estas intervenciones son de beneficio para las personas participantes.

De forma general, resultaría de interés que más estudios tomen en cuenta el estado inicial de las participantes, es decir, si presentan niveles elevados de ansiedad antes de tomar parte en el estudio. Como plantean Sheard y Maguire (1999), personas que ya presentan un diagnóstico de ansiedad pueden beneficiarse en mayor medida de intervenciones específicas para esta condición. En este sentido, tratamientos personalizados brindarían mejores resultados, al tomar en cuenta las necesidades iniciales y específicas de las participantes.

Asimismo, el seguimiento a las participantes, posteriormente a la finalización del tratamiento, es fundamental. Es necesario tener datos en relación con los efectos de las intervenciones y su duración. Con base en dicha información se toman en cuenta los tratamientos que produzcan resultados más prolongados en beneficio de la salud de las pacientes.

Por su parte, en cuanto a recomendaciones para investigaciones meta-analíticas, es necesario el uso añadido de fuentes no publicadas, con el objeto de disminuir el riesgo de sesgo. Como se planteó, las publicaciones pueden presentar el aspecto más favorable de los estudios, por lo que es necesaria la búsqueda de más información, con el fin de que los elementos considerados sean lo suficientemente comprehensivos.

Al considerar lo planteado hasta el momento, puede concluirse que las intervenciones psicológicas, como una totalidad, parecen tener resultados positivos en la reducción de los niveles de ansiedad en mujeres con cáncer de mama. El tamaño de efecto de estas intervenciones suele ser de pequeño a mediano y significativo, como se evidenció en esta investigación meta-analítica y en otros estudios similares a los que se hizo referencia. En este 
sentido, análisis posteriores podrían enfocarse en determinar qué variables contribuyen de manera específica a esta reducción, con el fin de potenciar los elementos terapéuticos para que los resultados de estas intervenciones sean más apreciables, beneficiosos y duraderos para las participantes.

\section{Referencias}

Ahmed, I., Sutton, A. J., \& Riley, R.D. (2012). Assessment of publication bias, selection bias, and unavailable data in meta-analyses using individual participant data: a database survey. British Medical Journal, 344, 1-10. doi: 10.1136/bmj.d7762.

Borenstein, M., Hedges, L. V., Higgins, J. P., \& Rothstein, H. R. (2009). Introduction to metaanalysis. United Kingdom: John Wiley \& Sons.

Botella, J., \& Gambara, H. (2002). Qué es el meta-análisis. Biblioteca Nueva: Madrid.

*Bredal, I., Kåresen, R., Smeby, N., Espe, R., Sørensen, E., Amundsen, M., ... \& Ekeberg, Ø. (2014). Effects of a Psychoeducational Versus a Support Group Intervention in Patients with Early-Stage Breast Cancer. Cancer Nursing, 37(3), $198-207$. doi: 10.1097/NCC.0b013e31829879a3.

Cramer, H., Lauche, R., Paul, A., \& Dobos, G. (2012). Mindfulness-based stress reduction for breast cancer- a systematic review and meta-analysis. Current Oncology,19(5), 343-352. doi: 10.3747/co.19.1016.

De la Torre-Luque, A., Gambara, H., López, E., \& Cruzado, J. (2016). Psychological treatments to improve quality of life in cancer contexts: A meta-analysis. International Journal of Clinical and Health Psychology, 16, 211-219.

*Dolbeault, S., Cayrou, S., Brédart, A., Viala, A., Desclaux, B., Saltel, P., ... \& Dickes, P. (2009). The effectiveness of a psycho-educational group after early-stage breast cancer treatment: results of a randomized French study. Psycho-Oncology, 18, 647656. doi: $10.1002 /$ pon. 1440

*Edmonds, C., Lockwood, G., \& Cunningham, A. (1999). Psychological Response to Long Term Group Therapy: A Randomized Trial with Metastatic Breast Cancer Patients. Psycho-Oncology I, 8(1), 74-91.

Faraone, S. (2008). Interpreting Estimates of Treatment Effects: Implications for Managed Care. Pharmacy and Therapeutics, 33(12), 700-711. doi: 10.12691/ijdsr-4-2-4

*Fernández, C., Villoria, E., Amigo, I., Padierna, C., Gracia, J., Fernández, R., \& Peláez, I. (2011). Terapia de activación conductual en pacientes con cáncer. Anales de Psicología, 27(2), 278-291.

*Gholamhosseini, S., \& Mojtabaie, M. (2014). Effectiveness of acceptance and commitment therapy (ACT) to reduce the symptoms of anxiety in women with breast cancer. International Journal of Review in Life Sciences, 4(1), 27-31.

Giménez, A. (2012). ¿Qué es un meta-análisis? y ¿cómo leerlo? Biomedicina, 7(1), 16-27.

Actualidades en Psicología, 34(128), 2020, 121-141 
*Groarke, A., Curtis, R., \& Kerin, M. (2013). Cognitive-behavioural stress management enhances adjustment in women with breast cancer. British Journal of Health Psychology, 18, 623- 641. doi: 10.1111/bjhp.12009

Hedges, L. V., \& Olkin, I. (1985). Statistical Methods for Meta-Analysis. London: Academic Press.

*Hoffman, C., Ersser, S., Hopkinson, J., Nicholls, P., Harrington, J., \& Thomas, P. (2012). Effectiveness of Mindfulness-Based Stress Reduction in Mood, Breast- and Endocrine-Related Quality of Life, and Well-Being in Stage 0 to III Breast Cancer: A Randomized, Controlled Trial. Journal of Clinical Oncology, 30(12), 13351342. doi: 10.1200/JCO.2010.34.0331

*Hopko, D., Armento, M., Robertson, S., Ryba, M., Carvalho, J., Colman, L., ... \& Lejuez, C. (2011). Brief Behavioral Activation and Problem-Solving Therapy for Depressed Breast Cancer Patients: Randomized Trial. Journal of Consulting and Clinical Psychology, 79(6), 834-849. doi: 10.1037/a0025450

Huang, J., \& Shi, L. (2016). The effectiveness of mindfulness-based stress reduction (MBSR) for survivors of breast cancer: study protocol for a randomized controlled trial. Trials, 17(209), 1-9. doi: 10.1186/s13063-016-1335-Z

Instituto Nacional de Estadística y Censos de Costa Rica. (2017). Estadísticas de defunciones generales 2016. Recuperado de http://www.inec.go.cr/sites/default/files/ documetos-biblioteca-virtual/repoblacevdefgendefinitivos2016.xlsx

Jadad, A., Moore, R., Carroll, D., Jenkinson, C., Reynolds, D., Gavaghan, D., \& McQuay, H. (1996). Assessing the Quality of Reports of Randomized Clinical Trials: IsBlinding Necessary? Controlled Clinical Trials, 17, 1-12. doi: 10.1016/01972456(95)00134-4

*Kim, Y., Kim, H., Ahn, S., Seo, Y., \& Kim, S. (2013). Effects of meditation on anxiety, depression, fatigue, and quality of life of women undergoing radiation therapy for breast cancer. Complementary Therapies in Medicine, 21, 379-387. doi: 10.1016/j. ctim.2013.06.005

Lim, C., Devi, M., \& Ang, E. (2011). Anxiety in women with breast cancer undergoing treatment: a systematic review. International Journal of Evidence-Based Healthcare, 9 , 215-235. doi: 10.1111/j.1744-1609.2011.00221.x

Littell, J., Corcoran, J., \& Pillai, V. (2008). Systematic Reviews and Meta-Analysis. NuevaYork: Oxford University Press.

*Loh, S., Packer, T., Tan, F., Xavier, M., Quek, K., \& Yip, C. (2012). Does a self management intervention lower distress in woman diagnosed with breast cancer? Japanese Psychological Research, 54(2), 159-169. doi: 10.1111/j.1468-5884.2011.00491.x

*Manne, S., Siegel, S., Heckman, C., \& Kashy, D. (2016). A Randomized Clinical Trial of a Supportive Versus a Skill-Based Couple-Focused Group Intervention for Breast Cancer Patients. Journal of Consulting and Clinical Psychology, 84(8), 668-681. doi: $10.1037 / \operatorname{ccp} 0000110$ 
Marín, F., Sánchez, J., \& López, J. (2009). El metaanálisis en el ámbito de las Ciencias de la Salud: una metodología imprescindible para la eficiente acumulación del conocimiento. Fisioterapia, 31(3), 107-114. doi: 10.1016/j.ft.2009.02.002

*Merckaert, I., Lewis, F., Delevallez, F., Herman, S., Caillier, M., Delvaux, N., ... \& Razavi, D. (2016). Improving anxiety regulation in patients with breast cancer at the beginning of the survivorship period: a randomized clinical trial comparing the benefits of single-component and multiple-component group interventions. Psycho-Oncology, 1-8. doi: 10.1002/pon.4294

Ministerio de Salud de Costa Rica. (2014). Situación Epidemiológica del Cáncer. Recuperado de http://www.ministeriodesalud.go.cr/index.php/vigilancia-de-la-salud/estadisticasy-bases-de-datos/estadisticas/estadistica-de-cancer-registro-nacional-tumores/2722situacion-epidemiologica-del-cancer/file

*Mohabbat-Bahar, S., Golzari, M., Moradi-Joo, M., \& Akbari, M. (2014). Efficacy of Group Logotherapy on Decreasing Anxiety in Women with Breast Cancer. Iranian Journal of Cancer Prevention, 7(3), 165-170.

*Mohabbat-Bahar, S., Maleki-Rizi, F., Akbari, M., \& Moradi-Joo, M. (2015). Effectiveness of Group Training Based on Acceptance and Commitment Therapy on Anxiety and Depression of Women with Breast Cancer. Iranian Journal of Cancer Prevention, $8(2), 71-76$.

Nelson, L., Simmons, J., \& Simonsohn, U. (2018). Psychology’s Renaissance. Annual Review of Psychology, 69, 511-534.

*Nunes, D. F., Rodriguez, A. L., da Silva Hoffmann, F., Luz, C., Filho, A. P., Muller M. C., Bauer, M. E. (2007). Relaxation and guided imagery program in patients with breast cancer undergoing radiotherapy is not associated with neuroimmunomodulatory effects. Journal of Psychosomatic Research, 63, 647-655. doi: 10.1016/j.jpsychores.2007.07.004

Organización Mundial de la Salud. (2020). Breast Cancer. Recuperado de https://www.who. int/cancer/prevention/diagnosis-screening/breast-cancer/en/

Rector, N., Bourdeau, D., Kitchen, K., \& Joseph-Massiah, L. (2008). Anxiety disorders: An information guide. Canadá: Centre for Addiction and Mental Health.

Richter, D., Koehler, M., Friedrich, M., Hilgendorf, I., Mehnert, A., \& Weißflog, G. (2015). Psychosocial interventions for adolescents and young adult cancer patients: A systematic review and meta-analysis. Critical Reviews in Oncology/Hematology, 95, 370-386. doi: 10.1016/j.critrevonc.2015.04.003

*Sarenmalm, E., Mårtensson, L., Andersson, B., Karlsson, P., \& Bergh, I. (2017). Mindfulness and its efficacy for psychological and biological responses in women with breast cancer. Cancer Medicine, 6(5), 1-15. doi: 10.1002/cam4.1052

Sheard, T., \& Maguire, P. (1999). The effect of psychological interventions on anxiety and depression in cancer patients: results of two meta-analyses. British Journal of Cancer, 80(11), 1770-1780. doi: 10.1038/sj.bjc.6690596 
Smith-Turchyn, J., Morgan, A., \& Richardson, J. (2016). The Effectiveness of Group-based Self-management Programmes to Improve Physical and Psychological Outcomes in Patients with Cancer: a Systematic Review and Meta-analysis of Randomised Controlled Trials. Clinical Oncology, 28, 292-305. doi: 10.1016/j.clon.2015.10.003

Tao, W., Jiang, P., Liu, Y., Aungsuroch, Y., \& Tao, X. (2015). Psycho-oncologic interventions to reduce distress in cancer patients: a meta-analysis of controlled clinical studies published in People's Republic of China. Psycho-Oncology, 24, 269-278. doi: 10.1002/pon.3634

Thomas, J. R., \& Nelson, J. K. (2007). Métodos de investigación en actividad física. Barcelona: Paidotribo.

*Würtzen, H., Dalton, S., Elsass, P., Sumbundu, A., Steding-Jensen, M., Karlsen, R., .. \& Johansen, C. (2013). Mindfulness significantly reduces self-reported levels of anxiety and depression: Results of a randomised controlled trial among 336 Danish women treated for stage I-III breast cancer. European Journal of Cancer, 49, 1365-1373. doi: 10.1016/j.ejca.2012.10.030

Yang, Y., Sui, G., Liu, G., Huang, D., Wang, S., \& Wang, L. (2014). The effects of psychological interventions on depression and anxiety among Chinese adults with cancer: a meta-analysis of randomized controlled studies. BMC Cancer, 14(956), 1-26. doi: 10.1186/1471-2407-14-956

Zhang, M., Wen, Y., Liu, W., Peng, L., Wu, X., \& Liu, Q. (2015). Effectiveness of Mindfulnessbased Therapy for Reducing Anxiety and Depression in Patients With Cancer: A Meta-analysis. Medicine, 94(45), 1-9. doi: 10.1097/MD.00000000000008

Zhang, J., Xu, R., Wang, B., \& Wang, J. (2016). Effects of mindfulness-based therapy for patients with breast cancer: A systematic review and meta-analysis. Complementary Therapies in Medicine, 26, 1-10. doi: 10.1016/j.ctim.2016.02.012 